each having a capacity of about 3000 cubic feet, while that of the bedrooms varies from 1700 to 1800 feet.

The whole of the south sides of the houses are furnished with ample verandahs, which, while they do not in the least obstruct the light or air, yet shelter the houses from the wind and rain, keep them cool in summer, and furnish on each story a convenient and agreeable promenade. It is intended, when all the houses are finished, to unite the several verandahs, enclosing them with sashes. In the winter a long sheltered walk of nearly 900 feet will thus be afforded, in which it will be practicable to walk in all weathers, and the means will also be afforded of getting to chapel the whole way under cover.

Although little over three years have elapsed since this hospital was first projected by me, I am happy to state that three pairs of houses are completed and furnished, two of them having for a considerable period been occupied with patients; the fourth pair, through the munificence of Sir William Malins, is in course of erection; the fifth pair will be shortly commenced; while, lastly, the building of the chapel is being proceeded with.

Under the guidance of the architect, T. Hellyer, Esq., of Ryde, great pains have been taken with the construction of the houses throughout to render them thoroughly dry and warm, light, airy, and cheerful. Neither paper nor paint has been used, and the walls have been made for the most part washable, while especial care has been bestowed upon the warming and ventilation. The system of warming adopted is by hot-water pipes, heated from one central furnace, one of these pipes being placed on each floor, and each pair of houses is provided with a hot bath. Each room, whether bed-room or sitting-room, is provided with inlet and outlet ventilators; the inlets are above and below, and the upper one is kept locked (so that it cannot be tampered with) at any degree of aperture which may be considered necessary, and which varies according to the season of the year. The ontlets are also below and above, and open into flues which go up to the top of the houses, and terminate in a horizontal shaft, which in its turn ends in one of the house flues. In this shaft a gas jet is placed whereby a traction power may be obtained, but which it is thought may be replaced hereafter by a hot-water coil. The passages are also carefully ventilated, and, while it is intended to burn gas, the air consumed will be obtained from without and the products of combustion will also be thus discharged, so that the atmosphere will be unaffected, in fact untouched.

I should mention that the hospital affords already accommodation to a house-surgeon and matron, with dispensary, library, and store-room; that there is in course of construction a mortuary, chapel, and post-mortem room while hereafter it is hoped that sufficient funds may be obtained for the erection of laundry, baths, and a central room, or hall, in which the patients, for purposes of amusement, from time to time, can assemble.

The least satisfactory part of the hospital, judged by the light of recent experience, is the sewerage. The sewers are tubular thronghout, and terminate in four cesspits at a distance from the houses. Two of these are lined with concrete, and are frequently emptied; the others are not at present, as they should be, rendered impermeable. It is intended specially to ventilate all the cesspits and to attach a pump to the two larger, so that the sewage can be pumped out from time to time and employed, if advisable, on the lands. The waste pipes also will all be disconnected.

Such is a very brief outline of the principal features of this hospital, which, I believe, is in some respects in advance of any hospital hitherto constructed on the separate or cottage principle; and I am happy to state that we have found it to realise in practice all the advantages contemplated.

The patients admitted within the walls express the greatest satisfaction with the arrangements, and recognise fully the many advantages of the separate system, and from some of them we have had some painful descriptions of the evils and trials, in cases of consumption and other affections of the organs of respiration, of the old ward system.

For an outlay of $\$ 32.000$ a thoroughly well-appointed hospital, on the latest and best principle, affording accommodation to over 100 in-patients, each with a separate bedroom, will be obtained, and comparing this outlay with that of other hospitals, and looking at the results obtained, the amount appears to me extremely moderate.

Though much has already been effected, more remains to be accomplished, and especially the General Committee are most solicitous to increase, by means of subscriptions, the annual and permanent income of the hospital.

I remain, Sir, your obedient servant,

Ventnor, February, 1872. ARthur Hirl Hassall, M.D.

\section{TYPHOID FEVER AND SEWER GAS.}

\section{To the Editor of THE LANCET.}

SiR,-I have been recently requested to report on the origin of two cases of typhoid fever in the family of the principal of a large school. This gentleman had six children, and two of them were attacked with typhoid fever in the early part of January, one fifteen and the other eighteen days after the breaking-up of the school for the holidays. The children occupied a suite of three rooms on the first floor. They are sufficiently large, and the air supply is wholesome. The last of the suite opened upon a corridor and staircase leading to the school bath-room and dormitories. The children were in charge of a nursemaid, and, with a remarkable exception, were treated and fed in the same way. This exception related to a habit acquired only by the affected children of drinking cold water freely, particularly at night; the younger ones have not been encouraged in the habit, but have been chiefly fed on milk, of which the supply was not stinted. The night supply of water was derived, for the sake of convenience, from a tap connected with two large cisterns supplying the baths, and from them the general supply for the boys' dormitories is also drawn. As this water had been used for months, it became necessary to examine the cistern more carefully, and to see what changes took place when the boys went home. The room in which the baths are placed has been divided by a partition reaching to the ceiling, and in the smaller part the cisterns were placed on cross beams high above the head. There was a small space left on one side, in which there is a window near the ceiling, and immediately beneath in the corner was a syphon watercloset. The tops of the cisterns were open, and it became obvious that any escape of sewer gas from the closet would be liable to be absorbed by the water, particularly if the window at the top were closed. During school term the window was usually kept open, and there was a constant demand for water. The closet, though in frequent use, was never observed to smell; indeed, it was continually flushed by the slops of the dormitories. But as soon as the boys broke up, the window and door of the closet were permanently closed, and the demand for water was greatly reduced as soon as the general scrubbing of the floors and corridors consequent on the dismissal of the boys had ceased. This involved an unusual pouring down of water through the closet. The syphon was thereby sucked dry, and the sewer gases, having full play upon the extensive surface of the water, were rapidly absorbed; indeed, when the door of the closet was for the first time opened after the children were struck down, the stench was quite unbearable, although there was no smell outside.

The source of the fever is thus made out, and we have direct proof of the poisoning of a large body of water by an exposure to sewer gases for the space of two or three days, and additional evidence that the period of incubation is from fourteen to sixteen days.

I am, Sir, yours, \&c.,

King's-road, Feb. 1872

J. H. STALLARD, M.B. Lond., \&c.

Testimonials. - Mr. John Pratt, surgeon, of Durham, was recently presented with a handsome gold watch and chain, and a purse of $\$ 16$. On the watch was the following inscription: "Presented by public subscription to Mr. John Pratt, Surgeon, for his unwearied exertions in relieving the poor during the prevalence of the smallpox epidenic in Durham and its neighbourhood. Durbam, 7th of February, 1872."-The Chillington Lodge, Ancient Order of Odd Fellows, Manchester Unity, at a meeting held in Wolverhampton on the 5th inst., presented their surgeon, Dr. F. Moses, with the sum of five guineas, in recognition of his services during the past quarter. 